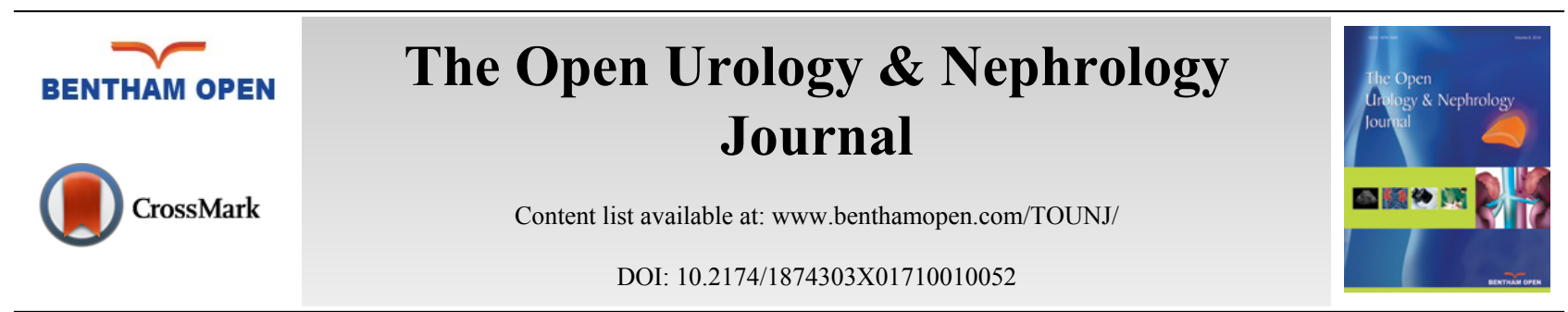

RESEARCH ARTICLE

\title{
Efficacy of Different Second-line Therapy Regimens in Metastatic Urothelial Carcinoma
}

Lukas Barwitz, Anne Berger, Stefanie Zschaebitz, Max Jenzer, Cathleen Nientiedt, Stefan Duensing, Dirk Jäger, Dogu Teber, Markus Hohenfellner and Carsten Grüllich ${ }^{*}$

Department of Oncology, University Heidelberg Oncology, Heidelberg, Germany

Received: August 15, 2017

Revised: November 23, 2017

Accepted: November 24, 2017

\section{Abstract:}

\section{Introduction:}

Metastatic Urothelial Cancer (UC) has a reported survival from platinum based chemotherapy of 15 months. Second line chemotherapy is considered relatively ineffective. Recently, new immuno-oncology drugs have been introduced.

\section{Objectives:}

Aim of this study was to analyze the survival by regimen and metastatic sites of second line treatment for UC.

\section{Methods:}

We analysed 70 patient receiving second line therapy between January 2010 and December 2016 at Heidelberg University Hospital. Median age was 60.9 years, male to female distribution was $74,3 \%$ to $25,7 \%$. Regimens used were vinflunine ( $\mathrm{n}=40,57,1 \%)$ taxane based $(n=20,28,6 \%)$ and immunotherapy $(n=9,12,9 \%)$.

Results:

Median overall survival (OS) from first line therapy over all lines was 28,0 months. Median OS from second line was 14,7 months (95\% CI, 11,4-18,0). No significant differences between regimens could be detected. OS of patients with lymphonodal only involvement ( $\mathrm{n}=16,22,5 \%)$ was 35.5 months (95\% CI 0.0-73.9), OS with visceral metastases excluding liver was 14.7 months $(95 \%$ CI 9.8-19.6) .and OS with any liver involvement was 9.4 months (95\% CI 0.0-20.9).

\section{Conclusion:}

Second line therapy for UC of selected patients leads to a prolonged survival compared to historical data. The choice of regimen appears not to influence OS. Lymphnodal only involvement is associated with the best prognosis.

Keywords: Urothelial cancer, Second line therapy, Vinflunine, Taxane, Immuno-oncology, Lymphonadal.

\section{INTRODUCTION}

Urothelial carcinomas are neoplasms of the urinary tract that arise in the bladder and, less commonly, in the renal pelvis, the ureter and the urethra. In the western world, transitional carcinoma is the predominant histologic subtype and accounts for approximately $90 \%$ of all cases. It is the 9 th most common cancer worldwide with approximately 430,000 new cases per year [1],leading to 165,000 annual deaths in Europe [2]. While urothelial carcinoma is very rare before the age of 40, incident rates increase with age and lead to a median age at diagnosis of 74 for men and 76 for women. For early disease, there is a curative approach including surgery and adjuvant chemotherapies. However, approximately

* Address correspondence to this author at the Department of Oncology, University Heidelberg Oncology, Heidelberg, Germany; Tel: 49(0)62215637125; E-mail: carsten.gruellich@med.uni-heidelberg.de 
$25 \%$ of patients initially present with metastatic disease and up to $50 \%$ develop them after cystectomy [3]. Hence, systemic palliative treatment is needed for many patients. With the most active, cisplatin-based treatment regimens, the median Overall Survival (OS) is reported with 15 months [4, 5]. Data concerning the efficacy of second-line chemotherapies are scarce, and only one phase III trial including 370 patients showed superiority of vinflunine over best supportive care (BSC)(6.9 versus 4.6 months).However, this trial did not reach statistical significance. Imbalances in prognostic factors between the treatment arms have led to controversial discussion of these results [6] Based on these results vinflunine is approved in Europe but not in the US [7]. Smaller phase II trial suggest some, though limited activity for single agent treatment with cytotoxic substances such as pemetrexed, taxanes [8 - 11] or oxaliplatin [12]. Recently, oncologic treatment options have widened substantially with the new field of Immune-Oncology (IO) also called immune-checkpoint inihibitors such as ipilimumab, pembrolizumab, nivolumab and atezolizumab, and those agents have shown promising activity in urothelial carcinoma [13 - 15]. Atezolizumab, a Programmed cell Death Ligand-1 (PDL-1) antibody and the Programmed cell Death 1 (PD-1) antibodies nivolumab and pembrolizumab were approved for treatment of patients refractory to platinum containing chemotherapy.

In our study, we retrospectively analyzed the outcome of second-line systemic treatment in patients with metastastic urothelial carcinoma with the aim of assessing overall survival from second line according to chosen regimen and survival across all lines. We further investigated the oncological outcomes according to metastatic sites involved. Lastly, we analyzed toxicities according to CTC criteria.

\section{PATIENTS AND METHODS}

We retrospectively analyzed patients who started palliative systemic treatment for Advanced Urothelial Carinoma (AUC) at our institution between January 2010 and December 2016. The data were obtained from the National Center for Tumor Diseases (Nationales Centrum für Tumorerkrankungen, NCT) Clinical Cancer Registry, a prospectively maintained database. The project was approved by the Heidelberg Ethics Committee (EKHD 0115). All patients had uniformly received a standard first-line therapy with either gemcitabin/cisplatin or Carboplatin for patients who weren't eligible for cisplatin, gemcitabin mono or a platin and taxan combination. Inclusion criteria for this analysis were the histological confirmation of urothelial carcinoma, metastatic or locally advanced, incurable disease and the start of a taxane, vinflunine or Immuno-oncology based second-line therapy regimen. The observation period for each patient started with initiation of second-line systemic treatment. The follow-up period for this analysis ended on 1st December 2016. Baseline patient characteristics were summarized using descriptive analysis.

\subsection{Assessment}

Clinical data were routinely collected and documented by the attending oncologists and medical staff via an electronic medical record. Information included Eastern Cooperative Oncology Group Performance Status (ECOG PS), start and stop date of systemic treatment, toxicities and consecutive dosage reductions of treatment, reasons for treatment interruptions, response to therapy, date of progression, and date of death. Side effects were registered according to the U.S. National Cancer Institute's Common Terminology Criteria for Adverse Events (CTCAE). Treatment was continued until clinically relevant or Response Evaluation Criteria in Solid Tumors (RECIST)-based radiographic progression, death, or intolerable toxicity occurred. Response was assessed by computed tomography or magnetic resonance imaging scans obtained according to the RECIST v1.1 criteria.

\subsection{Statistical Analysis}

Baseline patient characteristics were summarized using descriptive analysis. Overall Survival (OS) was calculated by Kaplan-Meier estimates. Log rank tests (Mantel-Cox) were used to compare OS across those groups; further tests were performed for subgroups according to different regimens and different metastatic sites. $p$ values of $<.05$ were considered significant. OS was calculated from the date of start of systemic treatment to the date of death or date of last follow-up (censored patients). Statistical analyses were conducted using the SPSS v21 software.

\section{RESULTS}

Of 841 patients with UC treated between January 2010 and December 2016 at the Urothelial Cancer Center of the NCT in Heidelberg 146 received first line treatment and of these 70 patients received a further second-line treatment. and were considered for this analysis. Of those 70 patients 24 patients received an additional third-line. Patient characteristics and therapies are summarized in Table $\mathbf{1}$. 
Table 1. Patient characteristics and therapies.

\begin{tabular}{|c|c|c|c|}
\hline Patient Characteristics & Diagnosis & 1st line & 2nd line \\
\hline No. of patients & 70 & 70 & 70 \\
\hline Age, years, median (range) & $\begin{array}{c}59,1 \\
(19-83)\end{array}$ & $\begin{array}{c}59,2 \\
(19-83)\end{array}$ & $\begin{array}{c}60,9 \\
(19-83) \\
\end{array}$ \\
\hline$<65(\%)$ & 4665,7 & 4665,7 & 4462,9 \\
\hline$>65(\%)$ & 2434,3 & 2434,3 & 27637,1 \\
\hline Gender, $\mathrm{M} / \mathrm{F}$ ratio & $52 / 18$ & $52 / 18$ & $52 / 18$ \\
\hline $\operatorname{ECOG}(0-1) /(2-3)$ & $70 / 0$ & $70 / 0$ & $61 / 9$ \\
\hline Metastatic disease & 41 & 65 & 70 \\
\hline \multicolumn{4}{|l|}{ Metastatic Site } \\
\hline LYM only $(\%)$ & $24(34,3)$ & $27(38,6)$ & $16(22,9)$ \\
\hline HEP $(\%)$ & $5(7,1)$ & $10(14,3)$ & $21(30)$ \\
\hline Visceral other than HEP (\%) & $7(10)$ & $28(40)$ & $33(47,1)$ \\
\hline Treatment discontinuation due to toxicity & & 2 & 4 \\
\hline
\end{tabular}

Abbreviations: ECOG 1/4 Eastern Cooperative Oncology Group; LYM lymphatic; HEP hepatic

\subsection{Patient Demographics}

Median age at the start of second-line treatment was 60,9 years, age distribution was 52 males $(74,3 \%)$ and 18 females $(25,7 \%)$. Overall patients presented in good condition measured by ECOG performance score at the beginning of second-line treatment. ECOG 0-1 performance scores were predominant in all second-line regimes' patients ( $\mathrm{n}=18$, $90,0 \%$ in taxane arm; $n=33,82,5 \%$ in vinflunine arm and $n=9,100,0 \%$ in Immuno arm) (Table 1). Distribution of the metastatic site at start of second-line treatment was visceral other than hepatic metastases $n=33(47.1 \%)$, any hepatic metastases $n=21(30 \%)$ and lymphonadal involvement only $n=16(22,9 \%)$ (Table 1). All detailed 2nd-line patient demographic information can be found in Table 2 .

\subsection{Chemotherapy and Toxicities}

The majority of patients received vinflunine as a second-line therapy $(n=40,57,1 \%)$. Other second-line therapies included a taxane based regime $(\mathrm{n}=20,28,6 \%)$ or Immunotherapy $(\mathrm{N}=9,12,9 \%)$ (Table 2$)$. In third-line patients received a platin based regime $(n=8,33,3 \%)$, a taxane based regime $(n=7,29,2 \%)$, vinflunine $(n=5,20,8 \%)$ or immunooncology ( $\mathrm{n}=4,16,7 \%)$ (Table 2$)$. Overall, treatments were well tolerated. Only 4 cases of treatment discontinuation due to toxicity were reported in second-line which occurred in two cases of taxane based (hematological failure, renal failure) and two cases of vinflunine based therapy (overall performance aggravation, hypertensive lapse). The main reason for drug discontinuation was progressive disease in $32(45,7 \%)$ patients in the second line and $13(54,2 \%)$ in the third line; and death in 8 patients in the second line $(11.4 \%)$ and $2(8,3 \%)$ in the third line. Adverse events measured by CTC (Common Toxicity Criteria) score were mainly lower (CTC 1-2) hematological ( $\mathrm{n}=29,41,4 \%)$, pain- $(\mathrm{n}=23,32,9)$ or nausea-related $(\mathrm{n}=17,24,3 \%)$ and could be found in all second-line regimes. There were four cases of grade III and two cases of grade IV hematological toxicity in taxane based regimes; and also one case of grade IV in vinflunine treated patients. Additional two cases of grade III diarrhea as well as two cases of grade III nausea were reported in immunotherapy treatments.

Table 2. Characteristics of second line treatment and adverse events.

\begin{tabular}{|c|c|c|c|c|c|}
\hline Characteristics & Taxane & Vinflunine & IO & Gem/Platin & Total \\
\hline No. of patients (\%) & 2028,6 & 4057,1 & 912,9 & 11,4 & 70 \\
\hline $\begin{array}{l}\text { OS median } \pm \mathrm{SD}^{1} \\
\text { (month) }\end{array}$ & $18,6 \pm 3,0$ & $12,3 \pm 0,2$ & $14,5 \pm 4,0$ & & $14,7 \pm 2,1$ \\
\hline \begin{tabular}{|l|}
$\begin{array}{l}\text { ECOG 0-1/2-3 } \\
(\%)\end{array}$ \\
\end{tabular} & $18 / 2(90 / 10)$ & $33 / 7(82,5 / 17,5)$ & $9 / 0(100 / 0)$ & $1 / 0(100 / 0)$ & $61 / 9(87,1 / 12,9)$ \\
\hline Age group 1/2 (\%) & $14 / 6(70 / 30)$ & $25 / 15(62,5 / 37,5)$ & $5 / 4(55,6 / 44,4)$ & $\begin{array}{c}0 / 1 \\
(0 / 100) \\
\end{array}$ & $44 / 26(62,9 / 37,1)$ \\
\hline Alive/deceased (\%) & $12 / 8(60 / 40)$ & $24 / 16(60 / 40)$ & $5 / 4(55,5 / 44,4)$ & $0 / 1$ & $41 / 29(58,6 / 41,4)$ \\
\hline Received 3rd line (\%) & $7(35)$ & $14(35)$ & $3(33,3)$ & $0(0)$ & $24(34,3)$ \\
\hline \multicolumn{6}{|l|}{ Metastatic Site } \\
\hline LYM only $(\%)$ & $7(35)$ & $7(17,5)$ & $2(22,2)$ & 0 & 16 \\
\hline
\end{tabular}


(Table प) contd.....

\begin{tabular}{|c|c|c|c|c|c|}
\hline Characteristics & Taxane & Vinflunine & IO & Gem/Platin & Total \\
\hline $\operatorname{HEP}(\%)$ & $5(25)$ & $11(27,5)$ & $5(55,5)$ & 0 & 21 \\
\hline Visceral other than HEP $(\%)$ & $8(40)$ & $22(55)$ & $2(22,2)$ & 1 & 33 \\
\hline \multicolumn{6}{|l|}{ Best remission } \\
\hline $\mathrm{CR}$ & 1 & 0 & 0 & 0 & 1 \\
\hline PR & 3 & 3 & 0 & 0 & 6 \\
\hline $\mathrm{SD}^{2}$ & 4 & 4 & 3 & 0 & 11 \\
\hline $\mathrm{PD}$ & 4 & 23 & 5 & 0 & 32 \\
\hline N/A & 8 & 10 & 2 & 1 & 20 \\
\hline \multicolumn{6}{|l|}{ No. of adverse events, all grades (CTC 3-4) } \\
\hline Hematological & $11(6)$ & $12(1)$ & $5(0)$ & $1(0)$ & $29(7)$ \\
\hline Nausea & $3(0)$ & $8(0)$ & $5(2)$ & $1(0)$ & $17(0)$ \\
\hline Vomiting & $1(0)$ & $2(0)$ & $1(0)$ & $1(0)$ & $5(0)$ \\
\hline Diarrhea & $1(0)$ & $1(0)$ & $3(2)$ & 0 & $5(2)$ \\
\hline Pain & $5(1)$ & $14(3)$ & $3(0)$ & $1(0)$ & $23(4)$ \\
\hline PNP & $4(0)$ & $1(0)$ & $2(0)$ & 0 & $7(0)$ \\
\hline Treatment discontinuation due to toxicity & 2 & 2 & 0 & 0 & 4 \\
\hline
\end{tabular}

Abbreviations: IO Immuno Oncology; Gem/Platin Gemctiabin/Platin; OS overall survival; ECOG 1/4 Eastern Cooperative Oncology Group; SD standard deviation; CR complete remission; PR part remission; $\mathrm{SD}^{2}$ stable disease; PD progressive disease; CTC Common Toxicity Criteria; PNP polyneuropathy

\subsection{Survival Analysis}

Overall Survival (OS) from the start of first line was 28,0 months (95\% confidence interval (CI), 17,5-38,5). (Fig. 1A, 1B) Median OS from the time of 2nd-line initiation was 14,7 months (95\% CI, 11,4-18,0) (Fig. 2A), taxane based regimes presented with an OS of 18,6 months $(95 \% \mathrm{CI}, 12,7-24,5)$; vinflunine based regimes with an OS of 12,3 months $(95 \%$ CI, 12,0-12,7) and immuno-oncology with an OS of 14,5 months $(95 \%$ CI, 6,5-22,4) and no statistically significant differences (Fig. 2B). Best response achieved in second-line was Complete Remission (CR) in 1 case of taxane (1,4\%), partial remission (PR) in 8,6\% (3 in taxane and 3 in vinflunine) and Stable Disease (SD) in 11,4\% (2 in taxane, 3 in vinflunine and 3 in immunotherapy) of patients. vinflunine (Table 1) Differences in survival could be observed depending on metastatic organ involvement. . While the median OS for patients with lymphonodal metastases only was 35,6 months $(95 \% \mathrm{CI}, 0,0-73,9)$, patients with hepatic metastases had a median OS of 9,4 months $(95 \% \mathrm{CI}$, $0,0-20,9)$ and patients with visceral metastases other than hepatic had a median OS of 14,7 months $(95 \%$ CI, 9,8-19,6). The difference between lymphonodal only and hepatic metastases was significant $(\mathrm{p}=0,025)$ as well as the difference between hepatic and visceral other than hepatic metastases ( $\mathrm{p}=0,035)$ (Fig. 2B).
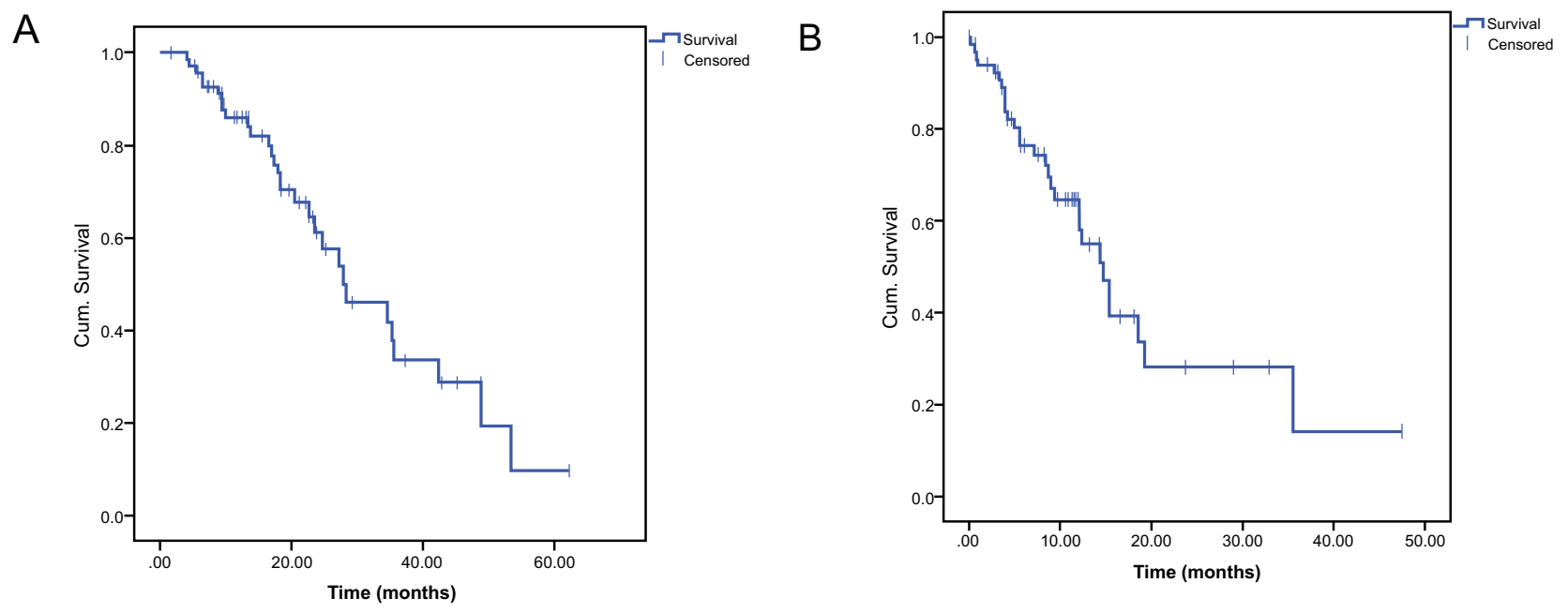

Fig. (1). Kaplan Meier curves for overall survival of all patients from first line treatment (A) and from second line treatment (B). 
A

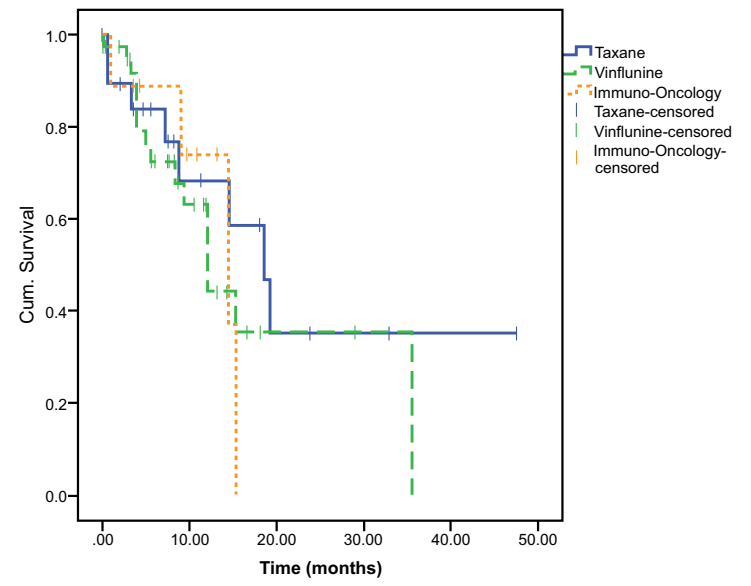

B

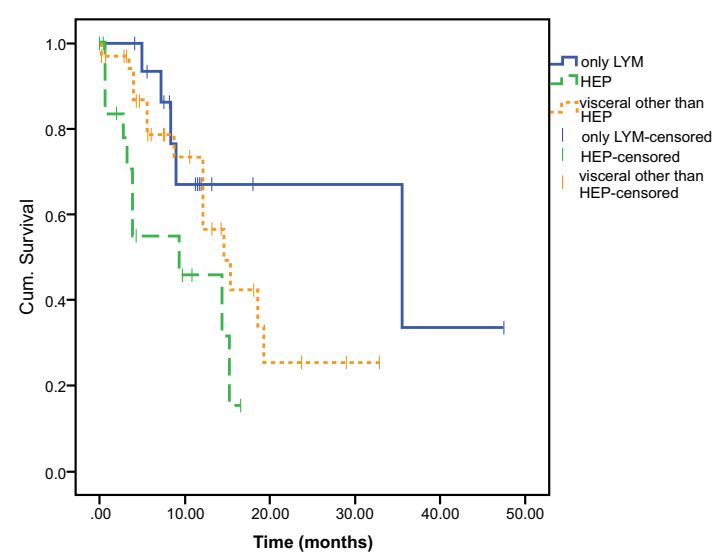

Fig. (2). Kaplan Meier curves for overall survival in second line according to treatment regimen (A) and according to metastatic spread (B).

\section{DISCUSSION}

Though UC is considered a chemo-sensitive disease literally all patients finally progress or relapse and die from the disease. As with all tumor diseases the most benefit can be achieved in first line treatment. Here, gemcitabine and cisplatin have largely replaced the more toxic combination of MVAC [16]. However, many patients are no candidates for cisplatin due to reduced renal function. For those patients carboplatin remains an option, although being less efficacious than cisplatin. As for second line after becoming refractory to platinum agents the evidence of clinical activity is low. Currently only vinflunine is approved for second in Europe but not in the U.S. due to a phase III trial that failed to reach statistical significance [7]. Further substances like taxenes and oxaliplatin have shown activity but only in small phase II trials [9, 10] and small retrospective series [17] Promising new agents are the anti-PD1 directed antibodies that showed significant activity in early trials. Lately, the first randomized phase III trial comparing anti-PD1 therapy against conventional chemotherapy has been presented and shows a statistically significant survival benefit [15]. The data presented in this study reflect the current praxis in second line. Most patients received vinflunine, the second largest group a taxane based regimen, while relatively few patients already were treated with anti-PD1 directed therapy, either within a clinical trial or following the approval of atezolizumab. For our patients we found a median survival of 14 months, which is much better than those reported in prospective trials and likely reflects a center effect of patient selection. In accordance with this we found mostly patients with favorable clinical performance to have received a second line therapy. It is well known that performance is the strongest predictor of OS for UC patients [18]. For the type of therapy chosen we could not detect any differences between vinflunine, taxanes or anti-PD1 immuno-oncology. As for immuno-oncology, the cohort is too small to detect differences to chemotherapy. However, non-inferiorty to chemotherapy is observed in this cohort, presumably with lower toxicity as reported by current trials [15]. As for chemotherapy these data appear to confirm the efficacy of a taxane based regimen compared to vinflunine without any superiority of either choice. When we analyzed the pattern of metastatic sites, lymphonodal metastatic spread only defined the best prognostic group as previously reported [19] compared to visceral spread, particularly hepatic spread showing the worst prognosis. Very few of our patients received a further $3^{\text {rd }}$ line and its use was balanced between different treatment groups, thus its influence on OS is limited. The overall toxicities were generally moderate (all grades: $n=86$ ) and mainly hematological or gastrointestinal. To assess the cumulative survival we analyzed the total OS across all lines of therapy. Here we found a cumulative OS of 28 months, which is much longer than expected from the published first line data and confirms the clinical meaningfulness of second line therapy for selected patients with metastatic UC. Limitations of our study are its relatively low numbers, the single center character and its retrospective nature. Hence, the data must be interpreted with the patient selection and large center effects in mind.

\section{CONCLUSION}

In conclusion selected patients with good performance status who fail after $1^{\text {st }}$ line platinum based therapy strongly 
benefit from second line therapy regardless of the regimen chosen and with acceptable toxicity. A median OS of over one year can be achieved with second line treatment for these patients. This results in a cumulative median OS across all of 28 months, which exceeds the historical data by far. The new immuno-oncology substances may well contribute to further extension of life expectancy for the majority of patients in the future.

\section{AUTHORS CONTRIBUTIONS}

L. Barwitz: Data collection and analysis, manuscript writing

A. Berger: Data collection, manuscript writing

S.Zschäbitz: Data collection

M. Jenzer: Data collection

C. Nientiedt: Data collection

S. Duensing: Data collection

D. Jäger: Data collection

D.Teber: Data collection

M. Hohenfellner: Data collection

C. Grüllich: Project development, Data collection and analysis, Manuscript writing and editing, Manuscript approval

\section{ETHICS APPROVAL AND CONSENT TO PARTICIPATE}

The project was approved by the Heidelberg Ethics Committee (EKHD 0115).

\section{HUMAN AND ANIMAL RIGHTS}

All procedures performed in studies involving human participants were in accordance with the ethical standards of the institutional and/or national research committee and with the 1964 Helsinki declaration and its later amendments or comparable ethical standards.

\section{CONSENT FOR PUBLICATION}

Not applicable.

\section{CONFLICT OF INTEREST}

The authors declare no conflict of interest, financial or otherwise.

\section{ACKNOWLEDGEMENTS}

The NCT is supported by the University Hospital of Heidelberg, in cooperation with the Medical Faculty Heidelberg, the German Cancer Research Center (DKFZ) and by the German Cancer Aid (Deutsche Krebshilfe).

\section{REFERENCES}

[1] Torre LA, Bray F, Siegel RL, Ferlay J, Lortet-Tieulent J, Jemal A. Global cancer statistics, 2012. CA Cancer J Clin 2015; 65(2): 87-108. [http://dx.doi.org/10.3322/caac.21262] [PMID: 25651787]

[2] Marcos-Gragera R, Mallone S, Kiemeney LA, et al. Urinary tract cancer survival in Europe 1999-2007: Results of the population-based study EUROCARE-5. Eur J Cancer 2015; 51(15): 2217-30. [http://dx.doi.org/10.1016/j.ejca.2015.07.028] [PMID: 26421824]

[3] Svatek RS, Siefker-Radtke A, Dinney CP. Management of metastatic urothelial cancer: The role of surgery as an adjunct to chemotherapy. Can Urol Assoc J 2009; 3(6)(Suppl. 4): S228-31. [PMID: 20019991]

[4] von der Maase H, Hansen SW, Roberts JT, et al. Gemcitabine and cisplatin versus methotrexate, vinblastine, doxorubicin, and cisplatin in advanced or metastatic bladder cancer: Results of a large, randomized, multinational, multicenter, phase III study. J Clin Oncol 2000; 18(17): 3068-77.

[http://dx.doi.org/10.1200/JCO.2000.18.17.3068] [PMID: 11001674] 
[5] Von der Maase H, Sengelov L, Roberts JT, et al. Long-term survival results of a randomized trial comparing gemcitabine plus cisplatin, with methotrexate, vinblastine, doxorubicin, plus cisplatin in patients with bladder cancer. J Clin Oncol 2005; 23(21): $4602-8$. [http://dx.doi.org/10.1200/JCO.2005.07.757] [PMID: 16034041]

[6] Ortmann CA, Mazhar D. Second-line systemic therapy for metastatic urothelial carcinoma of the bladder. Future Oncol 2013 ; $9(11)$ : $1637-51$. [http://dx.doi.org/10.2217/fon.13.139] [PMID: 24156324]

[7] Bellmunt J, Théodore C, Demkov T, et al. Phase III trial of vinflunine plus best supportive care compared with best supportive care alone after a platinum-containing regimen in patients with advanced transitional cell carcinoma of the urothelial tract. J Clin Oncol 2009; 27(27): 4454-61.

[http://dx.doi.org/10.1200/JCO.2008.20.5534] [PMID: 19687335]

[8] Vaughn DJ, Broome CM, Hussain M, Gutheil JC, Markowitz AB. Phase II trial of weekly paclitaxel in patients with previously treated advanced urothelial cancer. J Clin Oncol 2002; 20(4): 937-40. [http://dx.doi.org/10.1200/JCO.2002.20.4.937] [PMID: 11844814]

[9] McCaffrey JA, Hilton S, Mazumdar M, et al. Phase II trial of docetaxel in patients with advanced or metastatic transitional-cell carcinoma. J Clin Oncol 1997; 15(5): 1853-7. [http://dx.doi.org/10.1200/JCO.1997.15.5.1853] [PMID: 9164195]

[10] Meluch AA, Greco FA, Burris HA III, et al. Paclitaxel and gemcitabine chemotherapy for advanced transitional-cell carcinoma of the urothelial tract: a phase II trial of the Minnie pearl cancer research network. J Clin Oncol 2001; 19(12): 3018-24. [http://dx.doi.org/10.1200/JCO.2001.19.12.3018] [PMID: 11408496]

[11] Sweeney CJ, Roth BJ, Kabbinavar FF, et al. Phase II study of pemetrexed for second-line treatment of transitional cell cancer of the urothelium. J Clin Oncol 2006; 24(21): 3451-7. [http://dx.doi.org/10.1200/JCO.2005.03.6699] [PMID: 16849761]

[12] Winquist E, Vokes E, Moore MJ, Schumm LP, Hoving K, Stadler WM. A Phase II study of oxaliplatin in urothelial cancer. Urol Oncol 2005; 23(3): 150-4.

[http://dx.doi.org/10.1016/j.urolonc.2004.11.008] [PMID: 15907713]

[13] Rosenberg JE, Hoffman-Censits J, Powles T, et al. Atezolizumab in patients with locally advanced and metastatic urothelial carcinoma who have progressed following treatment with platinum-based chemotherapy: a single-arm, multicentre, phase 2 trial. Lancet 2016; 387(10031): 1909-20.

[http://dx.doi.org/10.1016/S0140-6736(16)00561-4] [PMID: 26952546]

[14] Morris VK, Salem ME, Nimeiri H, et al. Nivolumab for previously treated unresectable metastatic anal cancer (NCI9673): A multicentre, single-arm, phase 2 study. Lancet Oncol 2017; 18(4): 446-53.

[http://dx.doi.org/10.1016/S1470-2045(17)30104-3] [PMID: 28223062]

[15] Bellmunt J, de Wit R, Vaughn DJ, et al. Pembrolizumab as second-line therapy for advanced urothelial carcinoma. N Engl J Med 2017; 376(11): 1015-26. [http://dx.doi.org/10.1056/NEJMoa1613683] [PMID: 28212060]

[16] Sternberg CN, de Mulder PH, Schornagel JH, et al. Randomized phase III trial of high-dose-intensity methotrexate, vinblastine, doxorubicin, and cisplatin (MVAC) chemotherapy and recombinant human granulocyte colony-stimulating factor versus classic MVAC in advanced urothelial tract tumors: European organization for research and treatment of cancer protocol no. 30924. J Clin Oncol 2001; 19(10): 2638-46. [http://dx.doi.org/10.1200/JCO.2001.19.10.2638] [PMID: 11352955]

[17] Lee JH, Kang SG, Kim ST, et al. Modified MVAC as a second-line treatment for patients with metastatic Urothelial Carcinoma after Failure of Gemcitabine and Cisplatin Treatment. Cancer research and treatment : Official journal of Korean Cancer Association 2014; 46(2): $172-7$.

[18] Martinez-Salamanca JI, Shariat SF, Rodriguez JC, et al. Prognostic role of ECOG performance status in patients with urothelial carcinoma of the upper urinary tract: an international study. BJU Int 2012; 109(8): 1155-61. [http://dx.doi.org/10.1111/j.1464-410X.2011.10479.x] [PMID: 21883847]

[19] Sternberg CN, Skoneczna I, Kerst JM, et al. Immediate versus deferred chemotherapy after radical cystectomy in patients with pT3-pT4 or N+ M0 urothelial carcinoma of the bladder (EORTC 30994): An intergroup, open-label, randomised phase 3 trial. Lancet Oncol 2015; 16(1): 76-86.

[http://dx.doi.org/10.1016/S1470-2045(14)71160-X] [PMID: 25498218]

(C) Barwitz et al.

This is an open access article distributed under the terms of the Creative Commons Attribution 4.0 International Public License (CC-BY 4.0), a copy of which is available at: https://creativecommons.org/licenses/by/4.0/legalcode. This license permits unrestricted use, distribution, and reproduction in any medium, provided the original author and source are credited. 